\title{
The Role of Market Expansion on Equilibrium Bundling Strategies
}

\author{
Praveen K. Kopallea,*, Aradhna Krishna ${ }^{\mathrm{b}}$ and João L. Assunção ${ }^{\mathrm{c}}$ \\ a Amos Tuck School of Business Administration, Dartmouth College, Hanover, NH, USA \\ ${ }^{\mathrm{b}}$ Graduate School of Business, University of Michigan, Ann Arbor, MI, USA \\ ${ }^{\mathrm{c}}$ Universidade Catolica Portuguesa, Lisboa, Portugal
}

\begin{abstract}
Research on optimal bundling strategy has primarily dealt with the case of a monopolist and suggests that mixed bundling be adopted, as it allows for price discrimination. The overwhelming majority of consumer products, however, operate in a competitive arena, so that an adequate description of the bundling phenomenon needs to take account of alternative competitive product offerings. A few researchers have examined the duopolistic case-two suppliers each offering a bundle composed of two complementary products. However, the collective results do not paint a consistent picture. For example, Economides (1993. Mixed bundling in duopoly. Working Paper, Stern School of Business, New York University, EC-93-29) shows that the sub-game perfect Nash equilibrium bundling strategy is to offer a mixed bundle. By contrast, Anderson and Leruth (1993. Why firms may prefer not to price discriminate via mixed bundling. International Journal of Industrial Organization 11: 49-61), show that the solution is to offer pure components. The results of Matutes and Regibeau (1992. Compatibility and bundling of complementary goods in a duopoly. Journal of Industrial Economics 40: 37-54) suggest that the bundling strategy depends on consumer reservation price: mixed bundling when it is low and pure components when it is high. This paper offers an analytical analysis that reconciles these results by incorporating the moderating role of market expansion on equilibrium bundling strategies. Rendering comparable the conflicting results of such prior research requires selecting a methodology that not only sufficiently allows for their unique formal specifications, but which, in the current estimate, best captures empirical phenomena of broadest interest. The focus on market expansion suggests a model of the nested logit type (see Bucklin and Gupta 1992. Brand choice, purchase incidence, and segmentation: an integrated approach. Journal of Marketing Research 29: 201-215). It is shown that the sub-game perfect Nash equilibrium bundling strategy in a duopoly depends on the scope for market expansion, i.e., as the scope for market expansion decreases, the equilibrium bundling strategy shifts from mixed bundling to pure components. It is also shown that pure bundling will not be an equilibrium strategy. Finally, a discussion of the results when the assumption of perfect complementarity is relaxed is provided. Copyright (C) 1999 John Wiley \& Sons, Ltd.
\end{abstract}

\section{INTRODUCTION}

Product bundling, i.e., the sale of multiple products as a single package, is an increasingly popular brand management tactic. Examples abound for both durable and frequently purchased products: stereo receivers and speakers, washers and dryers, cameras and lenses, computers and monitors, hardware and software, cake mix and frost-

* Correspondence to: Amos Tuck School, Dartmouth College, 100 Tuck Hall, Hanover, NH 03755, USA. ing, etc. In these cases, three common strategy types, with varying degrees of reliance on bundling, have been identified (Adams and Yellen, 1976): (i) pure components (PC), where the goods are sold separately; (ii) pure bundling (PB), where the goods are sold only as a bundle; and mixed bundling (MB), where the goods are sold separately as well as together as a bundle.

Research on optimal bundling strategy has primarily dealt with the case of a monopolist (Adams and Yellen, 1976; Schmalensee 1982,

\section{Copyright (C) 1999 John Wiley \& Sons, Ltd.}


1984; McAfee et al., 1989; Braden, 1993), and suggests that mixed bundling (MB) be adopted as it allows for price discrimination. The overwhelming majority of consumer products, however, operate in a competitive arena, so that an adequate description of the bundling phenomenon needs to take account of alternative competitive product offerings (Jeuland, 1984). ${ }^{1}$ A few researchers have examined the duopolistic case-two suppliers each offering a bundle composed of two perfectly complementary products. However, the collective results do not paint a consistent picture. For example, Economides (1993) considers a twostage game where bundling strategy is decided in Stage 1 and prices in Stage 2. He shows that the sub-game perfect Nash equilibrium (SPNE) bundling strategy is to offer a mixed bundle. By contrast, Anderson and Leruth (1993), adopting a two-stage model as well, show that the SPNE solution is to offer pure components, as firms fear the extra degree of competition intrinsic to MB. Matutes and Regibeau (1992) consider a more complex game in which product compatibility comprises another stage. Given that compatibility prevails, they show that bundling strategy depends on consumer reservation price: MB when it is low and PC when it is high.

While Matutes and Regibeau's results show that both PC and MB can prevail under certain market conditions, a method for reconciling their results with those of Economides (1993) and of Anderson and Leruth (1993) does not immediately suggest itself. Rendering comparable the conflicting results of such prior research requires selecting a methodology that not only sufficiently allows for their unique formal specifications, but which, in our estimate, best captures empirical phenomena of broadest interest. A focus on market expansion suggests a model of the nested logit type (see Bucklin and Gupta, 1992), which can accommodate both saturated (i.e., little or no scope for market expansion) and unsaturated (i.e., considerable scope for market expansion) markets, by allowing consumers a 'no-purchase' option; such a model is an extension of the logit model appearing in Anderson and Leruth (1993). Higher consumer reservation price can be accommodated in the model via higher intrinsic product utility.

We use a nested logit specification, where low purchase incidence implies that the probability of making a purchase in the category is low. This suggests that a large proportion of consumers is not making any purchase at all. We define this as 'high scope for market expansion' since there is potential to increase the proportion of consumers making a purchase in the category. On the other hand, when the probability of purchase is high, we define this as 'low scope for market expansion' since most consumers are already making a purchase in the category. The modeling approach adopted here applies mature methodologies from the marketing literature on brand choice to enhance methods common to the economics literature on bundling. ${ }^{2}$

We demonstrate that the results from Matutes and Regibeau (1992), Anderson and Leruth (1993), and Economides (1993) are in fact quite consistent - that when the scope for market expansion is high, firms will follow MB; and when the scope for market expansion is low, firms will follow PC. Thus, we show that the SPNE bundling strategy in a duopoly depends on the scope for market expansion. As the scope for market expansion decreases, the equilibrium bundling strategy shifts from MB to PC. We also show that PB will not be an equilibrium strategy. Our results also hold under alternative assumptions about the error structure of our model specification.

Akin to Matutes and Regibeau (1992), Anderson and Leruth (1993), and Economides (1993), our model applies to two complementary products, where the use of one product requires the use of the other. For instance, consider a product pair of stereo receivers and speakers. A consumer making this purchase necessarily needs to buy both in order to derive any utility from this package. Other such complementary product pairs include washer and dryer, primer and paint, hardware and software, camera and lens, computer and monitor, etc. Our model does not apply to product bundles such as vacation packages, shampoos and conditioners, shirts and trousers, or crackers and cheese, where the use of one product does not necessitate the use of the other.

The rest of the paper is organized as follows. In the second section we discuss the consumer choice process for deciding among the available alternatives. In the third section, we present the firms' decision process to determine the equilibrium bundling strategy, given the consumer choice process. In the fourth section we present our results and in the fifth section we examine the sensitivity 
of our results to alternative consumer choice models and assumptions about the error distribution. Finally, we present a discussion of our results in the section 'Conclusions'.

\section{CONSUMER CHOICE PROCESS}

In this paper, we use a discrete choice logit framework to model consumer choice. Specifically, we use a nested logit specification, which is typically used to model consumer brand choice and purchase incidence (Ben-Akiva and Lerman, 1985; Bucklin and Lattin, 1991; Kannan and Wright 1991; Bucklin and Gupta, 1992). This model is a generalization of the logit model used in Anderson and Leruth (1993) by allowing a 'no purchase' option and hence incorporating the scope for market expansion. We assume two competing, symmetric firms, 1 and 2, both producing two complementary components, $x$ and $y$ (e.g., receiver and speakers). There exists compatibility among the firms' products, i.e., a component produced by one firm is compatible with the other component produced by either of the two firms. For notational simplicity, we denote the combined system consisting of $x$ and $y$ components as $(x, y)$ co-ordinates. For example, the system $(1,2)$ indicates that the $x$ component is manufactured by Firm 1 and the $y$ component is manufactured by Firm $2 .{ }^{3}$ Consumers make a purchase/no purchase decision and then, conditional on the purchase decision, consumers choose among the four systems, $(1,1),(1,2),(2,1)$ and $(2,2)$. Note that under the nested logit specification, consumers' 'purchase/no purchase' decision is indeed dependent on their evaluation of the attractiveness of each of the four systems. Thus, each consumer may buy one unit of each good (i.e., buying a system of the two complementary goods), or buy nothing. The structure of the model is given by Figure 1.

Consumer choice is modeled using nested multinomial logit. Conditional on purchase, consumer' utility for system $(i, j), i, j=1,2$, is denoted by $U_{i j}$, which is composed of the deterministic component $V_{i j}$ and error $\epsilon_{i j}$, which captures unobserved variations in tastes among the population. The deterministic component $V_{i j}$ is composed of the intrinsic utility for the system $\alpha_{i j}$, less the price, $p_{i j}$. Thus,

Copyright (C) 1999 John Wiley \& Sons, Ltd.

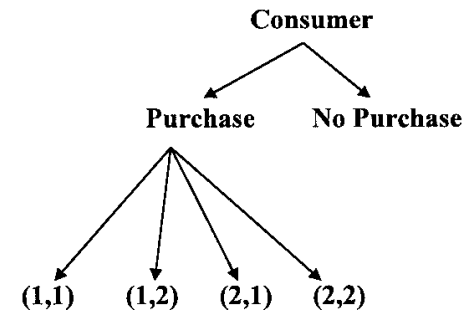

Figure 1. Consumer choice process: two-stage nested logit model.

$U_{i j}=V_{i j}+\epsilon_{i j}$

and

$V_{i j}=\alpha_{i j}-p_{i j}$

In the mental accounting literature that focuses on how consumers evaluate transactions, the model of Soman (1998) suggests that consumers make purchase decisions based on the 'net transaction value', which is the same as the deterministic component $\left(V_{i j}\right)$ of consumer utility in our model. Prelec and Loewenstein (1998) extend the notion of acquisition utility (i.e., the difference between intrinsic utility and price, which is identical to $V_{i j}$ ) put forth in Thaler (1985) and suggest that a consumer's purchase decision depends on the weighted difference between the positive intrinsic utility and the negative component, the price. The weighting depends on several situational factors, such as the timing of purchase versus the payment (Gourville and Soman, 1998). In the context of our model, it is assumed that the price payment for both goods comprising the bundle is made concurrently, and that the bundle is purchased (or acquired) at the same time as the payment is made. Hence, the acquisition utility for the bundle is $V_{i j}$. If, however, the two components are acquired (or paid for) at different times, or if the acquisition and payment of the bundle are temporally separated, the model needs to be modified accordingly.

Assuming equal intrinsic utility for each of the four systems, we have $\alpha_{11}=\alpha_{21}=\alpha_{12}=\alpha_{22}=\alpha$. The price of system $(i, j), p_{i j}$, is a function of the bundling strategy followed by the firm. If Firm $i$ practices $\mathrm{PC}, p_{i i}=p_{i .}+p_{. i}$, where $p_{i .}$ and $p_{. i}$ are the prices of components $x$ and $y$, respectively, offered by Firm $i$. If the firm practices MB or PB, the price for the bundle $(i, i)$ is denoted by $p_{i}^{b}$.

Assuming the error term $\epsilon_{i j}$ is independent, identical, and double exponentially distributed, 
following Ben-Akiva and Lerman (1985), demand for system $(i, j), D_{i j}$ is

$$
\begin{aligned}
& D_{i j}=\operatorname{Prob}(i, j) \\
& \quad=\operatorname{Prob}((i, j) \mid \text { purchase) Prob(purchase) } \\
& \operatorname{Prob}((i, j) \mid \text { purchase })=d_{i j \mid p}=\frac{\mathrm{e}^{V_{i j}}}{\sum_{j=1}^{2} \sum_{i=1}^{2} \mathrm{e}^{V_{i j}}}
\end{aligned}
$$

Prob $($ purchase $)=D=$

$$
\frac{\exp \left(\alpha_{B}+\theta_{B} \ln \left[\sum_{j=1}^{2} \sum_{i=1}^{2} \mathrm{e}^{V_{i j}}\right]\right)}{1+\exp \left(\alpha_{B}+\theta_{B} \ln \left[\sum_{j=1}^{2} \sum_{i=1}^{2} \mathrm{e}^{V_{i j}}\right]\right)}
$$

$\alpha_{B}$ reflects the intrinsic utility of a purchase and $\theta_{B}$ is the 'category value' that captures the attractiveness of the product category. In Anderson and Leruth's specification, demand for system $(i, j)$, $D_{i j}$, is given directly by Equation (4); this implies that the probability of purchase $(D)$ is exogenously set equal to 1 . On the other hand, the probability of purchase $(D)$ is endogenous in our model.

In a nested logit specification, Stage 1 denotes purchase incidence and Stage 2 is choice. Note that if $\alpha_{B}=0$ and $\theta_{B}=1$, the nested model specification takes the form of a joint logit model, where the option of not making any purchase is included as an additional alternative in the choice set. Since the nested logit model is a generalization of the joint logit model, we use the nested logit model. Both choice and purchase incidence are endogenous to our model since they depend on the specific prices charged by each firm. Low purchase incidence implies that the probability of making a purchase (denoted by $D$ in Equation (5)) in the category is low (and hence high scope for market expansion). On the other hand, when the probability of purchase $(D)$ is high, there is low scope for market expansion since most consumers are already making a purchase in the category. Note that the level of market saturation (and therefore the scope for market expansion) is endogenously determined in the nested logit model. The various parameters including the intrinsic utilities of systems $\left(\alpha_{i j}\right)$ and the intrinsic utility of a purchase $\left(\alpha_{B}\right)$, in conjunction with the corresponding price levels determine the level of market saturation. Anderson and Leruth's specification is a simpler logit model, which does not allow for the 'no-purchase' option, i.e., it assumes that everyone in the market makes a purchase in the category, i.e., a saturated market. Only when we replicate Anderson and Leruth's result, we remove the 'no-purchase' option, thus exogenously assuming market saturation.

In our model, a consumer might buy at most one unit of each of the two components. Note that when there is no limit on the number of units that a household may consume/buy, the 'scope for market expansion' needs to be modified accordingly by incorporating the purchase quantity decision as well. In this paper, we follow the single system purchase restriction of Matutes and Regibeau (1992) and Anderson and Leruth (1993).

\section{FIRMS' DECISIONS}

The marginal cost for producing components $x$ and $y$ are $c_{x}$ and $c_{y}$ respectively and are the same for the two firms (i.e., $c_{1 .}=c_{2 .}=c_{x}$ and $c_{.1}=c_{.2}=$ $c_{y}$ ). Following Matutes and Regibeau (1992), Anderson and Leruth (1993), and Economides (1993), we model the competition between the two firms as a two-stage game. In the first stage, firms choose the bundling strategy and in the second stage, they set prices. As mentioned in these prior papers, this scenario is representative of situations where the pricing decision is more easily reversible compared with the bundling decision, a reasonable assumption whenever bundling involves some changes in product design or packaging. Such examples include 'all in one' stereo centers, cameras with built-in lenses, computers with attached monitors, or factory installed option packages in the automobile industry (Matutes and Regibeau, 1992).

The three bundling strategies considered by the two firms are $\mathrm{PB}, \mathrm{PC}$, and $\mathrm{MB}$. This results in nine sub-games, namely, (PB, PB), (PB, PC), $(\mathrm{PB}, \mathrm{MB}), \quad(\mathrm{PC}, \mathrm{PB}), \quad(\mathrm{PC}, \mathrm{PC}), \quad(\mathrm{PC}, \mathrm{MB})$, $(\mathrm{MB}, \mathrm{PB}),(\mathrm{MB}, \mathrm{PC})$, and $(\mathrm{MB}, \mathrm{MB})$, where the first coordinate represents Firm 1's bundling strategy and the second coordinate represents Firm 2's bundling strategy. We use SPNE to determine the equilibrium bundling strategy. For each sub-game, the firms determine the Nash equilibrium prices and the corresponding profit. Based on the respective profits for the two firms in the nine sub-games, the SPNE bundling strategy is determined. Below, Lemma 1 shows that MB is at least as profitable as a PB strategy. 


\section{Lemma 1}

For $c_{x}, c_{y} \geq 0, i=1,2$, there exists an MB strategy such that PB is dominated. A PB strategy can be replaced by an $\mathrm{MB}$ strategy that is at least as profitable as the PB strategy. ${ }^{4}$

Proof

See Appendix A.

This result is similar to the result of Braden (1993) regarding the nonoptimality of pure bundling. We show that this extends to the more general setting of a nested logit framework. The logic of the proof is that if Firm 1 practices PB by offering the bundle at $p_{1}^{b}$, then the consumers who derive higher utility from $(1,1)$ would purchase the bundle $(1,1)$ at price $p_{1}^{b}$. Those consumers who get higher utility from $(1,2)$ (or from $(2,1)$ ) would buy the bundle $(1,1)$ at price $p_{1}^{b}$, but would also buy component $y$ (or component $x$ ) from Firm 2 and discard component $y$ (or component $x$ ) of the bundle $(1,1)$. We assume that there is no secondary market for such discarded goods. Thus, consumers who derive higher utility from $(1,2)$ (or from $(2,1)$ ) would be indifferent between buying component $x$ (or component $\mathrm{y}$ ) from Firm 1 at price $p_{1}^{b}$ and buying the bundle from Firm 1 at price $p_{1}^{b}$. Thus, if the firm adopts an MB strategy, offering the bundle at price $p_{1}^{b}$ and each component at price $p_{1}^{b}$, the firm's profit would be at least as high as in the PB strategy. If the components have a positive cost to Firm 1, then the firm will save on cost, thereby increasing profit. $^{5}$

Lemma 1 is consistent with research that has shown that $\mathrm{PB}$ at the very least will be weakly dominated by $\mathrm{MB}$ in a monopoly (McAfee et al., 1989) and in a single-product monopoly (Schmalensee, 1982; Lewbel, 1985). Lemma 1 shows that this result extends to a duopoly also.

\section{Optimal Prices and Profits for each Sub-game}

Lemma 1 reduces the nine sub-games to four, i.e., (PC, PC), (PC, MB), (MB, PC), and (MB, MB). We now characterize the Nash equilibrium prices for each of the four sub-games and determine the SPNE bundling strategy. The first-order conditions for the Nash equilibrium prices for sub-game $g$, where $g=(\mathrm{PC}, \mathrm{PC}), \quad(\mathrm{PC}, \mathrm{MB})$, $(\mathrm{MB}, \mathrm{MB})$, or $(\mathrm{MB}, \mathrm{PC})$, are obtained by differentiating $\pi_{i}^{g}$ for $i=1,2$ with respect to the prices in sub-game $g$ and equating them to zero. The equilibrium prices for sub-game (PC, PC) are derived in Appendix B. Equilibrium prices for other strategies are derived similarly. ${ }^{6}$ The maximum profit for Firm $i, i=1,2$, under sub-game $g$ is obtained by substituting the optimal prices in the respective profit function in sub-game $g$.

Sub-game 1. Pure Components by Both Firms.

When both firms follow PC, the profit for Firm $i$, $i=1,2$ is

$\pi_{i}^{\mathrm{PC}, \mathrm{PC}}=\left[\left(p_{i .}-c_{i .}\right)\left(D_{i i}+D_{i j}\right)+\left(p_{. i}-c_{. i}\right)\left(D_{i i}+D_{j i}\right)\right]$,

$j \neq i$

The equilibrium prices are given in Appendix B. Note that the prices, demand, profit, etc., under one bundling strategy may be different from those derived under another bundling strategy. For notational simplicity, we omit superscripts that would indicate the respective bundling strategy.

Sub-game 2. Both Firms follow Mixed Bundling.

When both firms follow an MB strategy the profit for Firm $i, i=1,2$, is

$$
\begin{aligned}
\pi_{i}^{\mathrm{MB}, \mathrm{MB}}= & {\left[\left(p_{i .}-c_{i .}\right) D_{i j}+\left(p_{. i}-c_{. i}\right) D_{j i}\right.} \\
& \left.+\left(p_{i}^{b}-c_{i .}-c_{. i}\right) D_{i i}\right], \quad j \neq i
\end{aligned}
$$

The equilibrium prices are derived as in the (PC, PC) case.

Sub-games 3 and 4. Firm $i$ follows Pure Components and Firm $j$ follows Mixed Bundling, $i, j=$ 1,$2 ; j \neq i$

If Firm $i$ offers PC and Firm $j$ offers MB, the profits for the two firms are given by

$\pi_{i}^{\mathrm{PC}, \mathrm{MB}}=\left[\left(p_{i .}-c_{i .}\right)\left(D_{i i}+D_{i j}\right)+\left(p_{. i}-c_{. i}\right)\left(D_{i i}+D_{j i}\right)\right]$,

$j \neq i$

$\pi_{j}^{\mathrm{PC}, \mathrm{MB}}=\left[\left(p_{j .}-c_{j .}\right) D_{j i}+\left(p_{. j}-c_{. j}\right) D_{i j}\right.$

$$
\left.+\left(p_{j}^{b}-c_{j .}-c_{. j}\right) D_{j j}\right], \quad j \neq i
$$

The equilibrium prices are derived as in the (PC, PC) case.

\section{Determination of Equilibrium Strategy}

Table 1 provides an overview of the firms' strategies in the sub-game framework. $\pi_{i}^{g}$ represents the

Manage. Decis. Econ. 20: 365-377 (1999) 
Table 1. Sub-game Framework

Firm 2

\begin{tabular}{llll} 
Firm 1 & Pure components & & Mixed bundling \\
\cline { 3 - 3 } & Pure components & $\pi_{1}^{\mathrm{PC}, \mathrm{PC}}, \pi_{2}^{\mathrm{PC}, \mathrm{PC}}$ & $\pi_{2}^{\mathrm{PC}, \mathrm{MB}}$ \\
\hline
\end{tabular}

profit of Firm $i, i=1,2$ for each sub-game $g$. We determine the non-cooperative Nash equilibrium strategy as follows.

(PC, PC) will be an SPNE solution if $\pi_{1}^{\mathrm{PC}, \mathrm{PC}}>\pi_{1}^{\mathrm{MB}, \mathrm{PC}}$ and $\pi_{2}^{\mathrm{PC}, \mathrm{PC}}>\pi_{2}^{\mathrm{PC}, \mathrm{MB}}$

Similarly, (PC, MB) will be an SPNE solution if $\pi_{1}^{\mathrm{PC}, \mathrm{MB}}>\pi_{1}^{\mathrm{MB}, \mathrm{MB}}$ and $\pi_{2}^{\mathrm{PC}, \mathrm{MB}}>\pi_{2}^{\mathrm{PC}, \mathrm{PC}}$

(MB, PC) will be an SPNE solution if $\pi_{1}^{\mathrm{MB}, \mathrm{PC}}>\pi_{1}^{\mathrm{PC}, \mathrm{PC}}$ and $\pi_{2}^{\mathrm{MB}, \mathrm{PC}}>\pi_{2}^{\mathrm{MB}, \mathrm{MB}}$

Finally, (MB, MB) will be an SPNE solution if $\pi_{1}^{\mathrm{MB}, \mathrm{MB}}>\pi_{1}^{\mathrm{PC}, \mathrm{MB}}$ and $\pi_{2}^{\mathrm{MB}, \mathrm{MB}}>\pi_{2}^{\mathrm{MB}, \mathrm{PC}}$

In the next section, we present the model propositions.

\section{MODEL PROPOSITIONS}

Model propositions are derived numerically by varying the values of marginal cost $\left(c_{x}\right.$ and $\left.c_{y}\right)$ in the range $0-1$ in steps of 0.25 , intrinsic utility for a system $\left(\alpha_{i j}\right)$ from 0 to 6 in steps of 0.25 , intrinsic utility of a purchase $\left(\alpha_{B}\right)$ from 0 to 3 in steps of 0.25 , and the impact of category attractiveness $\left(\theta_{B}\right)$ from 0.1 to 0.9 in steps of 0.1 . We assume equal intrinsic utility for all four systems, i.e., $\alpha_{11}=\alpha_{21}=\alpha_{12}=\alpha_{22}=\alpha$. The values for $\alpha_{i j}, \alpha_{B}$, and $\theta_{B}$ were chosen based on prior empirical research on logit models of brand choice and purchase incidence (Bucklin and Lattin, 1991; Chintagunta et al., 1991; Kannan and Wright, 1991; Bucklin and Gupta, 1992; Fader et al., 1992). Note that, ceteris paribus, the lower the intrinsic utility for a system $\left(\alpha_{i j}\right)$ or the intrinsic utility of a purchase $\left(\alpha_{B}\right)$, the higher the scope for market expansion, i.e., the lower the probability of purchase, $D$. The following propositions were robust across all simulation values.

Copyright (C) 1999 John Wiley \& Sons, Ltd.

\section{Proposition 1}

The SPNE bundling strategy in a duopoly depends on the scope for market expansion. As the scope for market expansion decreases, the equilibrium bundling strategy shifts from MB to PC.

When there is considerable scope for market expansion (for example, when the intrinsic utility for a system $\left(\alpha_{i j}\right)$ is low or the intrinsic utility of a purchase $\left(\alpha_{B}\right)$ is low), the payoff structure is similar to a prisoner's dilemma where both firms would be better off if they both offer PC. However, each firm in the duopoly has a unilateral incentive to increase the demand for its products by offering an MB. Thus, the SPNE strategy is that both firms offer MB (where the bundle price is lower than the sum of the prices of the two individual components) to induce consumers who are not buying any system to buy their system and hence increase the demand. Consequently, if both firms are offering PC, then each firm benefits by unilaterally switching to $\mathrm{MB}$ and the equilibrium strategy is (MB, MB).

On the other hand, when there is little scope for market expansion (for example, higher levels of intrinsic utility for a system $\left(\alpha_{i j}\right)$ and/or higher levels of intrinsic utility of a purchase $\left.\left(\alpha_{B}\right)\right)$, a decrease in the bundle price of system $(i, i), i=1$, 2 , in an MB strategy does not increase demand for it significantly since the market is already saturated. Although it attracts consumers who are buying the bundle from the other firm it will decrease the profit obtained from consumers who are purchasing the system $(i, i)$ anyway. Thus, each firm does not have a unilateral incentive to switch from PC to MB when there is no scope for market expansion.

To illustrate Proposition 1, we increase the scope for market expansion by varying consumers' intrinsic utility of a purchase $\left(\alpha_{B}\right)$. Higher levels of $\alpha_{B}$ will result in lower levels for scope of market expansion, i.e., increased market saturation. We obtain the same result when $\alpha_{i j}$ is varied. 
We vary $\alpha_{B}$ in the range $[0,3]$ in steps of 0.25 , with the following values for the other parameters: $\quad c_{x}=c_{y}=0, \quad \alpha_{11}=\alpha_{21}=\alpha_{12}=\alpha_{22}=0 \quad$ and $\theta_{B}=0.8$. Figure 2 illustrates this proposition.

When the intrinsic utility of a purchase $\left(\alpha_{B}\right)$ is low, there is considerable scope for market expansion, given the other parameter values. Of course, the exact level of market saturation, $D$, depends on the values of all parameters and each firm's bundling and pricing policies. Ceteris paribus, a lower value of $\alpha_{B}$ will result in a lower market saturation level than for a higher value of $\alpha_{B}$. When $\alpha_{B}=1.5$ and both firms follow PC, the equilibrium prices are $p_{1 .}=p_{1 .}=p_{2 .}=p_{.2}=1.27$ and the corresponding profits are $\pi_{1}=\pi_{2}=0.81$. When Firm $i$ follows PC and Firm $j$ follows MB, $i, j=1,2 ; j \neq i$, we get prices $p_{i .}=p_{. i}=1.00, p_{j .}=$ $p_{. j}=p_{j}^{b}=1.93$ and profits, $\pi_{1}=0.66, \pi_{2}=0.85$. When both firms follow $\mathrm{MB}, p_{1 .}=p_{.1}=p_{2 .}=$ $p_{.2}=p_{1}^{b}=p_{2}^{b}=1.75$, and profits, $\pi_{1}=\pi_{2}=0.69$. Across all four sub-games the probability of purchase, $D$, ranges from 0.64 to 0.68 . We find that the SPNE solution is, for both firms, to follow MB.

When we increase $\alpha_{B}$ to 3.0 , there is little scope for market expansion. In this case, when both firms follow PC, the profits are 1.31 each. When Firm 1 follows PC and Firm 2 follows MB, the

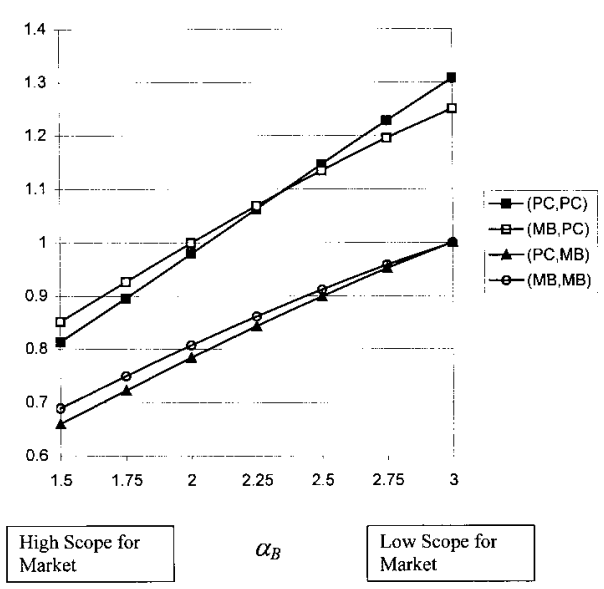

Figure 2. Impact of market expansion on bundling strategy. $\alpha_{B}$ denotes the intrinsic utility of a purchase; higher values of $\alpha_{B}$ imply lower scope for market expansion. (MB, PC) denotes that Firm 1 follows MB and Firm 2 follows PC. The same interpretation holds for (PC, PC), (PC, MB), and (MB, MB). profits are, respectively, 1.0 and 1.25 (symmetric for MB, PC). When both firms follow MB, each firm gets a profit of 1.0. Thus, the SPNE solution is, for both firms, to follow PC. The probability of purchase ranges from 0.83 to 0.88 .

We obtain a similar result when $\alpha_{11}=\alpha_{21}=$ $\alpha_{12}=\alpha_{22}=\alpha$ is varied from 0 to 6 and $\alpha_{B}=0$. When $\alpha$ is low, MB is the equilibrium bundling strategy and when $\alpha$ is high (say 6.0), the equilibrium bundling strategy is PC. Thus, the above analysis indicates that as the scope for market expansion decreases the equilibrium bundling strategy shifts from MB to PC.

Anderson and Leruth (1993) do not include the option of not making a purchase in their consumer choice model, i.e., the probability of purchase $(D)$ is 1, implying no scope for market expansion. To replicate this in our model, we eliminate the no-purchase decision by setting the probability of purchase $(D)$ equal to 1 . Hence, the conditional probability of purchasing a system is equal to the unconditional probability of purchase. We find the following:

\section{Proposition 2}

When consumers do not have the option of not making a purchase, i.e., when there no scope for market expansion, PC is the SPNE bundling strategy for each firm.

This result actually follows from Proposition 1 and replicates the finding of Anderson and Leruth (1993).

\section{SENSITIVITY TO ERROR DISTRIBUTION}

In our model we assumed independently and identically distributed (iid) error terms for the four systems. In this section, we show that our propositions are invariant to two other error structures, namely

(i) the error terms are different for own systems $((1,1)$ and $(2,2))$ and combined systems $((1,2)$ and $(2,1))$ - we model this error structure using a three-stage nested logit approach;

and

(ii) there is correlation in error terms between systems sharing a component, e.g., $(1,1)$ and $(1,2)$ - we model this using a probit approach (Ben-Akiva and Lerman, 1985).

Manage. Decis. Econ. 20: 365-377 (1999) 


\section{(i) Different Error Terms for Own Systems and Combined Systems}

We assume the iid error terms for own systems are drawn from a different distribution than those for combined systems. We use a three-stage nested logit model to determine the equilibrium bundling strategy. The structure of this model is given in Figure 3.

While the three-stage nested logit model has been used before (Ben-Akiva and Lerman, 1985; Kannan and Wright, 1991), its use in a bundling context is novel. Derivation of the model is given in Appendix C. To determine the equilibrium bundling strategy, we conducted a numerical analysis using the three-stage nested logit model (Appendix C). The results suggest that although the prices are different under each bundling strategy, the equilibrium bundling strategies remain the same, i.e., our results in the previous section still hold. Therefore, in this paper, we focus on the simpler, two-stage nested logit specification.

\section{(ii) Correlation in Error Terms between Systems Sharing a Component}

Correlation in error terms between systems sharing a component cannot be modeled using a nested logit model since it does not allow a system to be in more than one branch. For example, if we were to model correlation in error terms between systems sharing a component, systems $(1,1)$, $(1,2)$, and $(2,1)$ should be in one branch, and systems $(2,2),(1,2)$, and $(2,1)$ in another branch. We therefore use a probit model (Ben-Akiva and Lerman, 1985) and modify the specification to allow for a 'no-purchase' option. While probit models have been used in the marketing literature for brand choice decisions, these have typically not included a choice of 'no-purchase'. The derivation of the model is given in Appendix D.

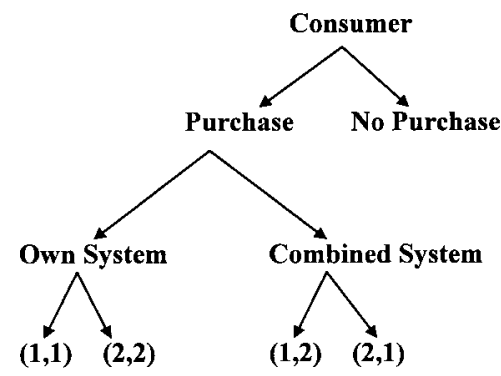

Figure 3. Consumer choice process: three-stage nested logit model.
We conducted a set of simulations using the modified probit model. We assume zero correlation between systems that do not share a component, and a correlation of 0.5 between systems that share a component. Again, we find that our results hold. Although the probit specification allows us to develop the profit function for each bundling strategy, it is not tractable enough to derive the equilibrium prices. Hence, we focus on the simpler logit model in this paper.

\section{CONCLUSIONS}

This paper extends the literature on product bundling in a duopoly by examining the role of market expansion on equilibrium bundling strategy. We first show that PB will not be an equilibrium strategy. We then demonstrate that the SPNE bundling strategy depends on the scope for market expansion. As the scope for market expansion decreases, the equilibrium bundling strategy shifts from MB to PC. Thus, we demonstrate that the results from Matutes and Regibeau (1992), Anderson and Leruth (1993), and Economides (1993) are in fact quite consistent.

Explanations for our propositions are intuitively appealing. When there is considerable scope for market expansion, each firm has a unilateral incentive to offer a mixed bundle such that the price for the bundle is lower than the sum of the prices of the individual components. This results in (MB, MB) as an SPNE bundling strategy. The equilibrium strategy switches from (MB, MB) to (PC, PC) when the market becomes more saturated (i.e., when there is little or no scope for market expansion) because each duopolist lacks the unilateral incentive to offer a MB, since it involves offering both the components at a price that is lower than the sum of the two prices in a PC strategy. Each firm has to offer a large price cut to make consumers switch from a system they inherently prefer to another system. In doing so, the firms lose a significant profit from their existing consumer base. Thus, offering a bundle at a reduced price is no longer profitable. Hence, when the market is saturated or close to saturation, (PC, PC) is the equilibrium bundling strategy.

In this paper, we assume that the two goods are complementary, i.e., the purchase of one good necessitates the purchase of the other. There are, 


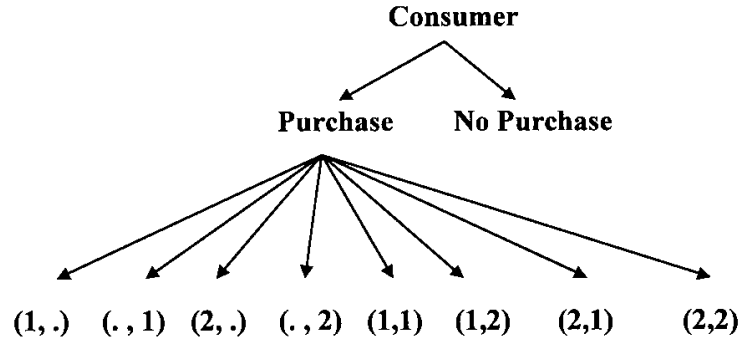

Figure 4. Consumer choice process: component and system purchase.

however, many situations where this does not apply; for example, concert tickets, vacation packages, etc. In such cases, the appropriate two-stage nested logit model is given in Figure 4.

The purchase decision is made in Stage 1 and in Stage 2 consumers make a purchase from the following set of alternatives: $x_{1}, x_{2}, y_{1}, y_{2},\left(x_{1}, x_{2}\right)$, $\left(x_{1}, y_{2}\right),\left(y_{1}, x_{2}\right),\left(y_{1}, y_{2}\right)$. We thus extend our model to include the above eight alternatives. ${ }^{7}$ Let $\gamma_{1}$ and $\gamma_{2}$. denote the intrinsic utilities for Component $x$ made by Firms 1 and 2, respectively; similarly, let $\gamma_{1}, \gamma_{2}$ be the corresponding intrinsic utilities for Component $y$. The intrinsic utility for a system, say $(1,2)$, is then given by $\gamma_{1}+\gamma_{.2}$. For simplicity, we assume $\gamma_{1 .}=\gamma_{2 .}=\gamma_{.1}=\gamma_{.2}=\gamma$. We varied the intrinsic utility for a component $(\gamma)$ from 0 to 6 in steps of 0.25 . We find that as the intrinsic utility for a component $(\gamma)$ increases, the equilibrium bundling strategy shifts from MB to PC. When the intrinsic utility for a component is high, firms have to offer a deeper price cut under MB to induce switching to their system. However, the firms would then be losing margin from those who would have purchased their system any way. Hence, we see PC. When the intrinsic utility for a component $(\gamma)$ is low, each firm has an incentive to offer a mixed bundle in order to attract not only those consumers who do not make any purchase (for example, when the intrinsic utility of a purchase, $\alpha_{B}$, is low) but also to attract those consumers who buy single components and induce them to buy both components from that firm. Thus, when consumers are able to derive utility upon purchasing a unit of either component, the equilibrium firm behavior depends on the intrinsic utility of a component; $\mathrm{MB}$ when it is low and PC when it is high. This result is consistent with what we find in the case of perfect complements.
Our model has implications for segmented markets with different levels of market saturation. Taking foreign and domestic markets as one case of segmented markets, the model implies that when firms enter a foreign market, they need not practice the same bundling strategy as in the home market. One reason for entering a foreign market could be that the home market is saturated, whereas the foreign market is not. In such a case, PC may be the equilibrium bundling strategy in the home market and $\mathrm{MB}$ in the foreign market.

Finally, we also examine the effect of relaxing some of our model assumptions on the equilibrium bundling strategy. Specifically, in the second section we assumed iid error terms for the four systems. A sensitivity analysis of our propositions to changes in the error distribution shows that although the prices are different under each bundling strategy, the equilibrium bundling strategies are the same in the two other error structures that we consider.

This research can be extended in a number of directions. In this paper, we focus on a duopoly; future research could investigate if our propositions extend to an oligopoly. In addition, it would be interesting to consider cases where there are more than two components that could be offered in a bundle. Also, following the standard approach in the economics literature (Matutes and Regibeau, 1992; Anderson and Leruth, 1993; Economides, 1993; Chen, 1997), we adopted a two-stage game for the bundling and pricing decisions. However, this is indeed an assumption in our modeling framework since other games could lead to different solutions. Future research could examine the sensitivity of our results to the type of game employed. Yet another direction for future research is to allow consumers to purchase more than one unit of either component and examine the corresponding equilibrium bundling strategy. The objective of this paper is to offer an analytical analysis of the role of market expansion on equilibrium bundling strategies. A worthwhile extension would be to validate the model assumptions and the results using either experimental or 'real' data.

\section{Acknowledgements}

The authors would like to thank the editor, two anonymous reviewers, Fred Feinberg, Don Lehmann, and Scott Neslin for their comments on earlier drafts of this manuscript. 


\section{APPENDIX A}

\section{Proof of Lemma 1}

By contradiction. Let PB be an optimal strategy for Firm 1 where $p_{1}^{* b}$ is the optimal price of the bundle. Let the price for Firm 2's Component $y$ be $p_{.2}$ and for Component $x$ be $p_{2 .}$. Let $\pi_{1}^{*}$ be the profit for Firm 1, and $D_{11}^{*}, D_{12}^{*}, D_{21}^{*}$ be the corresponding unconditional purchase probabilities for systems $(1,1),(1,2)$, and $(2,1)$, respectively. Thus, when Firm 1 follows PB, the profit for Firm 1 is

$\left(p_{1}^{* b}-c_{1 .}-c_{.1}\right)\left(D_{11}^{*}+D_{12}^{*}+D_{21}^{*}\right)=\left(p_{1}^{* b}-c_{1 .}-c_{.1}\right) D_{11}^{*}+\left(p_{1}^{* b}-c_{1 .}-c_{.1}\right) D_{12}^{*}+\left(p_{1}^{* b}-c_{1 .}-c_{.1}\right) D_{21}^{*}$

If, on the other hand, Firm 1 follows MB such that the price of each of the individual components is equal to the price of the bundle under PB, the profit for Firm 1 is

$\left(p_{1}^{* b}-c_{1 .}-c_{.1}\right) D_{11}^{*}+\left(p_{1}^{* b}-c_{1 .}\right) D_{12}^{*}+\left(p_{1}^{* b}-c_{.1}\right) D_{21}^{*}$

However, for $c_{1 .}, c_{.1}>0$, the profit from PB for Firm 1 is less than the profit from MB, since

$\left(p_{1}^{* b}-c_{1 .}-c_{.1}\right) D_{11}^{*}+\left(p_{1}^{* b}-c_{1 .}-c_{.1}\right) D_{12}^{*}+\left(p_{1}^{* b}-c_{1 .}-c_{.1}\right) D_{21}^{*}$

$<\left(p_{1}^{* b}-c_{1 .}-c_{.1}\right) D_{11}^{*}+\left(p_{1}^{* b}-c_{1}\right) D_{12}^{*}+\left(p_{1}^{* b}-c_{.1}\right) D_{21}^{*}$

Further, when $c_{1 .}=c_{.1}=0$, the profit from $\mathrm{MB}$ is at least as high as the profit obtained from following a PB strategy.

\section{APPENDIX B}

\section{Deriving Equilibrium Prices for the (PC, PC) Sub-game in a Duopoly}

The first-order conditions are given by

$\frac{\partial \pi_{i}^{\mathrm{PC}, \mathrm{PC}}}{\partial p_{i .}}=0, \quad i=1,2$

$\frac{\partial \pi_{i}^{\mathrm{PC}, \mathrm{PC}}}{\partial p_{. i}}=0, \quad i=1,2$

Solving the first-order conditions and simplifying, we obtain the following the equilibrium prices:

$p_{i .}=c_{i .}$

$$
+\frac{D_{i i}+D_{i j}+\left(p_{. i}-c_{. i}\right)\left[\left(d_{i i \mid D}+d_{i j \mid D}\right)\left(D_{i i}+D_{j i}+D_{j i} \theta_{B} D-\theta_{B}\left(D_{i i}-D_{j i}\right)\right)-D_{i i} D \theta_{B}\left(d_{i j \mid D}-d_{i i \mid D}\right)-D_{i i}\right]}{\left(d_{i i \mid D}+d_{i j \mid D}\right)\left(D_{i i} D \theta_{B}+D_{i i} \theta_{\mathrm{B}}+d_{i j} \theta_{\mathrm{B}}-D_{i i}-D_{i j}-d_{i j \mid D} D \theta_{\mathrm{B}}\right)+D_{i i}+D_{i j}}
$$

$p_{. i}=c_{. i}$

$$
+\frac{D_{i i}+D_{j i}+\left(p_{i .}-c_{i .}\right)\left[\left(d_{i||}+d_{j i \mid D}\right)\left(D_{i i}+D_{i j}+D_{i j} \theta_{B} D-\theta_{B}\left(D_{i i}-D_{i j}\right)\right)-D_{i i} D \theta_{B}\left(d_{j i \mid D}-d_{i i \mid D}\right)-D_{i i}\right]}{\left(d_{i i \mid D}+d_{j i \mid}\right)\left(D_{i i} D \theta_{B}+D_{i i} \theta_{B}+d_{j i} \theta_{B}-D_{i i}-D_{j i}-d_{j i \mid} D \theta_{B}\right)+D_{i i}+D_{j i}}
$$

where $i, j=1,2 ; j \neq i$.

\section{APPENDIX C}

\section{Derivation of a Three-stage Nested Logit Model}

Following Ben-Akiva and Lerman (1985), the demand for the system $(i, i), \mathrm{i}=1,2$, is

$D_{i i}=P(i, i)=P((i, i) \mid$ own $\&$ purchase $) P($ own $\mid$ purchase $) P($ purchase $)$

$P((i, i) \mid$ own \& purchase $)=\frac{\mathrm{e}^{V_{i i}}}{\mathrm{e}^{V_{i i}}+\mathrm{e}^{V_{j j}}}$

Copyright (C) 1999 John Wiley \& Sons, Ltd.

Manage. Decis. Econ. 20: 365-377 (1999) 
$P($ own $\mid$ purchase $)=\frac{\exp \left[\alpha_{\mathrm{os}}+\theta_{\mathrm{oc}} \ln \left(\mathrm{e}^{V_{i i}}+\mathrm{e}^{V_{j j}}\right)\right]}{\exp \left[\alpha_{\mathrm{os}}+\theta_{\mathrm{oc}} \ln \left(\mathrm{e}^{V_{i i}}+\mathrm{e}^{V_{j j}}\right)\right]+\exp \left[\alpha_{\mathrm{cs}}+\theta_{\mathrm{oc}} \ln \left(\mathrm{e}^{V_{i j}}+\mathrm{e}^{V_{j i}}\right)\right]}$

$P($ purchase $)=\frac{\exp \left\{\alpha_{B}+\theta_{B} \ln \left[\exp \left(\alpha_{\mathrm{os}}+\theta_{\mathrm{oc}} \ln \left(\mathrm{e}^{V_{i i}}+\mathrm{e}^{V_{j j}}\right)\right)+\exp \left(\alpha_{\mathrm{cs}}+\theta_{\mathrm{oc}} \ln \left(\mathrm{e}^{V_{i j}}+\mathrm{e}^{V_{j i}}\right)\right)\right]\right\}}{1+\exp \left\{\alpha_{B}+\theta_{B} \ln \left[\exp \left(\alpha_{\mathrm{os}}+\theta_{\mathrm{oc}} \ln \left(\mathrm{e}^{V_{i i}}+\mathrm{e}^{V_{j i}}\right)\right)+\exp \left(\alpha_{\mathrm{cs}}+\theta_{\mathrm{oc}} \ln \left(\mathrm{e}^{V_{i j}}+\mathrm{e}^{V_{j i}}\right)\right)\right]\right\}}$

$\alpha_{B}$ reflects the intrinsic utility of purchase and $\theta_{B}$ is the category value. $\alpha_{\mathrm{os}}$ and $\alpha_{\mathrm{cs}}$ are the intrinsic utilities from purchasing an own system and a combined system, respectively. $\theta_{\text {oc }}$ captures the varying attractiveness of own systems versus the combined systems.

Similarly, demand for system $(i, j), i, j=1,2 ; j \neq i$, is

$D_{i j}=P(i, j)=P((i, j) \mid$ combined \& purchase $) P($ combined $\mid$ purchase $) P($ purchase $)$

$P((i, j) \mid$ combined \& purchase $)=\frac{\mathrm{e}^{V_{i j}}}{\mathrm{e}^{V_{i j}}+\mathrm{e}^{V_{j i}}}$

$P($ combined $\mid$ purchase $)=\frac{\exp \left[\alpha_{\mathrm{cs}}+\theta_{\mathrm{oc}} \ln \left(\mathrm{e}^{V_{i j}}+\mathrm{e}^{V_{j i}}\right)\right]}{\exp \left[\alpha_{\mathrm{os}}+\theta_{\mathrm{oc}} \ln \left(\mathrm{e}^{V_{i i}}+\mathrm{e}^{V_{j j}}\right)\right]+\exp \left[\alpha_{\mathrm{cs}}+\theta_{\mathrm{oc}} \ln \left(\mathrm{e}^{V_{i j}}+\mathrm{e}^{V_{j i}}\right)\right]}$

\section{Simulations for the Three-stage Nested Logit Model}

We assume $\alpha_{\mathrm{os}}=\alpha_{\mathrm{cs}}=0$ and vary the set of values for $\theta_{B}$ and $\theta_{\mathrm{oc}}$ in the interval [0.1,0.9] in steps of 0.1 . We vary the values of marginal cost $\left(c_{x}\right.$ and $\left.c_{y}\right)$ in the range $0-1$ in steps of 0.25 , intrinsic utility for a system $\left(\alpha_{i j}\right)$ from 0 to 6 in steps of 0.25 , and intrinsic utility of a purchase $\left(\alpha_{B}\right)$ from 0 to 3 in steps of 0.25 .

\section{APPENDIX D}

\section{Derivation of Modified Probit Model}

The probit model assumes that $\epsilon_{i j} \sim N\left[0, \sigma_{i j}\right]$. Below, we modify the standard multinomial probit model in order to include the 'no-purchase' option. We first obtain the conditional probability of purchasing a system $(i, j)$ and then multiply it with the probability of purchase and thus obtain the unconditional probability of purchasing system $(i, j)$.

The conditional probability of purchase for system $(i, i)$ is given by

$P(i, i)=P\left(U_{i i}>0, U_{i i}>U_{i j}, U_{i i}>U_{j i}, U_{i i}>U_{j j}\right)$

where $i, j=1,2 ; j \neq i$.

Let $\sigma_{i i}^{2}, \sigma_{j j}^{2}, \sigma_{i j}^{2}$, and $\sigma_{j i}^{2}$, denote the variance of $e_{i i}, e_{j j}$, $e_{i j}$, and $e_{j i}$, respectively. Similarly, let $\sigma_{i i, i j}^{2}$ denote the covariance between $e_{i i}$ and $e_{i j}$, and let $\sigma_{i i, j i}^{2}$ denote the covariance between $e_{i i}$ and $e_{j i}$. Replacing $U_{i j}$ by $V_{i j}+\epsilon_{i j} \forall i, j$, moving the error terms on the left side and observed utilities on the right, and dividing the resulting inequalities by the variance of the respective left-hand side expressions, this translates to

$$
\begin{aligned}
P(i, i)= & P\left(\frac{e_{i i}}{\sigma_{i i}}>\frac{-v_{i i}}{\sigma_{i i}}, \frac{e_{i i}-e_{j j}}{\sqrt{\sigma_{i i}^{2}+\sigma_{j j}^{2}-2 \sigma_{i i, j j}}}>\frac{v_{j j}-v_{i i}}{\sqrt{\sigma_{i i}^{2}+\sigma_{j j}^{2}-2 \sigma_{i i, j j}}}, \frac{e_{i i}-e_{j i}}{\sqrt{\sigma_{i i}^{2}+\sigma_{j i}^{2}-2 \sigma_{i i, j i}}}\right. \\
& \left.>\frac{v_{j i}-v_{i i}}{\sqrt{\sigma_{i i}^{2}+\sigma_{j i}^{2}-2 \sigma_{i i, j i}}}, \frac{e_{i i}-e_{i j}}{\sqrt{\sigma_{i i}^{2}+\sigma_{i j}^{2}-2 \sigma_{i i, i j}}}>\frac{v_{i j}-v_{i i}}{\sqrt{\sigma_{i i}^{2}+\sigma_{i j}^{2}-2 \sigma_{i i, i j}}}\right)
\end{aligned}
$$

Let the standardized terms

$\frac{e_{i i}}{\sigma_{i i}}, \quad \frac{e_{i i}-e_{j j}}{\sqrt{\sigma_{i i}^{2}+\sigma_{j j}^{2}-2 \sigma_{i i}, j j}}, \quad \frac{e_{i i}-e_{j i}}{\sqrt{\sigma_{i i}^{2}+\sigma_{j i}^{2}-2 \sigma_{i i, j i}}}, \quad$ and $\frac{e_{i i}-e_{i j}}{\sqrt{\sigma_{i i}^{2}+\sigma_{j i}^{2}-2 \sigma_{i i, i j}}}$

be denoted by $z_{1}, z_{2}, z_{3}$, and $z_{4}$, respectively. Thus, we have

Copyright (C) 1999 John Wiley \& Sons, Ltd.

Manage. Decis. Econ. 20: 365-377 (1999) 
$P(i, i)=P\left(z_{1}>\frac{-v_{i i}}{\sigma_{i i}}, z_{2}>\frac{v_{j j}-v_{i i}}{\sqrt{\sigma_{i i}^{2}+\sigma_{j j}^{2}-2 \sigma_{i i, j j}}}, z_{3}>\frac{v_{j i}-v_{i i}}{\sqrt{\sigma_{i i}^{2}+\sigma_{j i}^{2}-2 \sigma_{i i, j i}}}, z_{4}>\frac{v_{i j}-v_{i i}}{\sqrt{\sigma_{i i}^{2}+\sigma_{i j}^{2}-2 \sigma_{i i, i j}}}\right)$

Let $\Phi\left(z_{1}, z_{2}, z_{3}, z_{4}\right)$ denote standard multivariate normal distribution, i.e.,

$\Phi\left(z_{1}, z_{2}, z_{3}, z_{4}\right)=\frac{1}{(2 \Pi)^{p / 2}(|R|)^{1 / 2}} \mathrm{e}^{-Z^{\prime} R^{-1} Z}$

where $Z^{\prime}$ is the vector given by $\left[\begin{array}{llll}z_{1} & z_{2} & z_{3} & z_{4}\end{array}\right], R$ is the $4 \times 4$ correlation matrix of $z_{1}, z_{2}, z_{3}$, and $z_{4}, p$ is the rank $R$, and $|R|$ is the determinant of $R$. For example, the correlation between $\mathrm{z}_{1}$ and $\mathrm{z}_{2}$ is given by

\section{Covariance $\left(z_{1}, z_{2}\right)$}

$\overline{\left(\text { Standard Deviation }\left(z_{1}\right)\right)\left(\text { Standard Deviation }\left(z_{2}\right)\right)}$

i.e.,

$\frac{\sigma_{11}^{2}-\sigma_{11} \sigma_{22}}{\sigma_{11} \sqrt{\sigma_{11}^{2}+\sigma_{22}^{2}-2 \sigma_{11,22}^{2}}}$

Finally, we have,

$$
\begin{aligned}
P(i, i)= & \int_{-v_{i i} / \sigma_{i i}}^{\infty} \int_{\left(v_{j j}-v_{i i}\right) / \sqrt{\sigma_{i l}^{2}+\sigma_{j j}^{2}-2 \sigma_{i i, j j}}}^{\infty} \int_{\left(v_{j i}-v_{i i)}\right) / \sqrt{\sigma_{i l}^{2}+\sigma_{j i}^{2}-2 \sigma_{i i, j i}}}^{\infty} \int_{\left(v_{i j}-v_{i i)}\right) / \sqrt{\sigma_{i l}^{2}+\sigma_{i j}^{2}-2 \sigma_{i i, i j}}}^{\infty} \\
& \times \Phi\left(z_{1}, z_{2}, z_{3}, z_{4}\right) \mathrm{d} z_{4} \mathrm{~d} z_{3} \mathrm{~d} z_{2} \mathrm{~d} z_{1}
\end{aligned}
$$

Conditional probabilites of systems $(i, j),(j, i)$ and $(j, j)$ are derived similarly.

The demand of each system, i.e., the unconditional probability of purchase of system $(i, i)$ is obtained by multiplying the conditional probability of purchasing system $(i, i)$ with the probability of purchase. The probability of purchase is given by

$$
\begin{aligned}
P(\text { purchase }) & =1-P\left(U_{11}<0, U_{12}<0, U_{21}<0, U_{22}<0\right) \\
& =1-P\left(e_{11}<-V_{11}, e_{12}<-V_{12}, e_{21}<-V_{21}, e_{22}<-V_{22}\right) \\
& =1-P\left(\frac{e_{11}}{\sigma_{11}}<\frac{-V_{11}}{\sigma_{11}}, \frac{e_{12}}{\sigma_{12}}<\frac{-V_{12}}{\sigma_{12}}, \frac{e_{21}}{\sigma_{21}}<\frac{-V_{21}}{\sigma_{21}}, \frac{e_{22}}{\sigma_{22}}<\frac{-V_{22}}{\sigma_{22}}\right)
\end{aligned}
$$

Let the standardized terms, $e_{11} / \sigma_{11}, e_{12} / \sigma_{12}, e_{21} / \sigma_{21}$, and $e_{22} / \sigma_{22}$, be denoted by $x_{1}, x_{2}, x_{3}$, and $x_{4}$, respectively. Thus, we have

$P($ purchase $)=P\left(x_{1}<\frac{-V_{11}}{\sigma_{11}}, x_{2}<\frac{-V_{12}}{\sigma_{12}}, x_{3}<\frac{-V_{21}}{\sigma_{21}}, x_{4}<\frac{-V_{22}}{\sigma_{22}}\right)$

Let $\Phi\left(x_{1}, x_{2}, x_{3}, x_{4}\right)$ denote standard multivariate normal distribution, i.e.,

$\Phi\left(x_{1}, x_{2}, x_{3}, x_{4}\right)=\frac{1}{(2 \Pi)^{q / 2}(|S|)^{1 / 2}} \mathrm{e}^{-X^{\prime} S^{-1} X}$

where $X^{\prime}$ is the vector given by $\left[\begin{array}{llll}x_{1} & x_{2} & x_{3} & x_{4}\end{array}\right], S$ is the $4 \times 4$ correlation matrix of $x_{1}, x_{2}, x_{3}$, and $x_{4}, q$ is the rank $S$, and $|S|$ is the determinant of $S$. For example, the correlation between $x_{1}$ and $x_{2}$ is given by $\sigma_{11,12}^{2} / \sigma_{11} \sigma_{22}$. Thus, we have

$P($ purchase $)=1-\int_{-\infty}^{-v_{i i} / \sigma_{i i}} \int_{-\infty}^{-v_{i j} / \sigma_{i j}} \int_{-\infty}^{-v_{j i} / \sigma_{j i}} \int_{-\infty}^{-v_{j j} / \sigma_{j j}} \Phi\left(x_{1}, x_{2}, x_{3}, x_{4}\right) \mathrm{d} x_{4} \mathrm{~d} x_{3} \mathrm{~d} x_{2} \mathrm{~d} x_{1}$

\section{NOTES}

1. Chen (1997) focuses on a duopoly in one product market and perfect competition in the other. Using a two-stage game, where the duopolists first decide

Copyright (C) 1999 John Wiley \& Sons, Ltd. whether to produce one good or both goods and then decide the optimal prices, Chen (1997) shows that the sub-game perfect equilibrium is for one firm to produce only one good and the other firm to offer a pure bundle.

Manage. Decis. Econ. 20: 365-377 (1999) 
2. Note that this paper also extends the marketing literature on product bundling, which has primarily focused on the exploration of behavioral issues concerned with bundling (Drumwright, 1992; Majumdar and Jun, 1993; Yadav and Monroe, 1993; Yadav, 1994), comparison of different bundling options (Guiltinan, 1987), approaches for pricing of bundles (Hanson and Martin, 1990; Venkatesh and Mahajan, 1993), and a discussion of conditions that justify 'unbundling' (Wilson et al., 1990).

3. Although the product pairs we consider are complementary, the various systems may not be perfectly substitutable, i.e., while $x_{1}$ may be used with $y_{2}$, the system $(2,2)$ may be preferable to $(1,2)$ depending upon consumers' intrinsic utility for each of the two systems.

4. For $c_{x}, c_{y}>0, \mathrm{MB}$ will be strictly more profitable than PB. For $c_{x}=c_{y}=0, \mathrm{MB}$ will be at least as profitable as PB.

5. Consistent with Matutes and Regibeau (1992) and Anderson and Leruth (1993), it is assumed there are no cost advantages in PB.

6. The derivations are available from the authors upon request.

7. The complete derivations are available from the authors and follow along the lines of the nested logit model in the second section of this paper.

\section{REFERENCES}

Adams WJ, Yellen JL. 1976. Commodity bundling and the burden of monopoly. Quarterly Journal of Economics 90: 475-498.

Anderson SP, Leruth L. 1993. Why firms may prefer not to price discriminate via mixed bundling. International Journal of Industrial Organization 11: $49-61$.

Braden DJ. 1993. Bundling with competitively priced goods. Working Paper, University of Rochester, Rochester, NY.

Ben-Akiva M, Lerman SR. 1985. Discrete Choice Analysis. MIT Press: Cambridge, MA.

Bucklin RE, Gupta S. 1992. Brand choice, purchase incidence, and segmentation: an integrated approach. Journal of Marketing Research 29: 201-215.

Bucklin RE, Lattin JM. 1991. A two-state model of purchase incidence and brand choice. Marketing Science 10: 24-39.

Chen Y. 1997. Equilibrium product bundling. Journal of Business 70(1): 85.

Chintagunta PK, Jain DC, Vilcassim NJ. 1991. Investigating heterogeneity in brand preferences in logit models for panel data. Journal of Marketing Research 28: $417-429$.

Drumwright M. 1992. A demonstration of anomalies in evaluations of bundling. Marketing Letters 3: 311321.
Economides N. 1993. Mixed bundling in duopoly. Working Paper, New York University, Stern School of Business, EC-93-29.

Fader PS, Lattin JM, Little JDC. 1992. Estimating nonlinear parameters in the multinomial logit model. Marketing Science 11: 372-385.

Gourville JT, Soman D. 1998. Payment depreciation: the behavioral effects of temporally separating payments from consumption. Journal of Consumer Research 25: 160-174.

Guiltinan JP. 1987. The price bundling of services: a normative framework. Journal Of Marketing 51: 7485.

Hanson W, Martin RK. 1990. Optimal bundle pricing. Management Science 2: 155-176.

Jeuland A. 1984. Comments on Gaussian demand and commodity bundling. Journal of Business 57(1): S231-S233.

Kannan PK, Wright GP. 1991. Modeling and testing structured markets: a nested logit approach. Marketing Science 10: 58-82.

Lewbel A. 1985. Bundling of substitutes or complements. International Journal of Industrial Organization 3: 101-107.

Matutes C, Regibeau P. 1992. Compatibility and bundling of complementary goods in a duopoly. Journal of Industrial Economics 40: 37-54.

Majumdar T, Jun SY. 1993. Consumer evaluation of multiple versus single price changes. Journal of Consumer Research 20(3): 441-450.

McAfee RP, McMillan J, Whinston MD. 1989. Multiproduct monopoly, commodity bundling and correlation of values. Quarterly Journal of Economics 104: 371-383.

Prelec D, Loewenstein G. 1998. The red and the black: mental accounting of savings and debt. Marketing Science 17(1): 4-28.

Schmalensee R. 1982. Commodity bundling by singleproduct monopolies. Journal of Law and Economics 25: $67-71$.

Schmalensee R. 1984. Gaussian demand and commodity bundling. Journal of Business 57(1): S211-S230.

Soman D. 1998. The illusion of delayed incentives: evaluation future effort-money transactions. Journal of Marketing Research 35: 427-437.

Thaler R. 1985. Mental accounting and consumer choice. Marketing Science 4(3): 199-214.

Venkatesh R, Mahajan V. 1993. A probabilistic approach to pricing a bundle of products or services. Journal of Marketing Research 30: 494-508.

Wilson LO, Weiss AM, John G. 1990. Unbundling of industrial systems. Journal of Marketing Research 27: 123-138.

Yadav MS. 1994. How buyers evaluate product bundles: a model of anchoring and adjustment. Journal of Consumer Research 21(2): 342-353.

Yadav MS, Monroe KB. 1993. How buyers perceive savings in a bundle price: an examination of a bundles' transaction value. Journal of Marketing Research 30: 350-358. 